\title{
Attitudes and beliefs of healthcare workers about influenza vaccination
}

\author{
Carmel Halpin, Bernie Reid
}

Citation

Halpin C, Reid B (2019) Attitudes and beliefs of healthcare workers about influenza vaccination. Nursing Older People. doi: 10.7748/nop.2019.e1154

\author{
Peer review

\section{Correspondence} \\ bb.reid@ulster.ac.uk \\ Conflict of interest \\ None declared \\ Accepted \\ 14 January 2019 \\ Published online \\ February 2019
}

This article has been subject to external double-blind peer review and has been checked for plagiarism using automated software

\begin{abstract}
Background Annual influenza vaccination is recommended for all healthcare workers (HCWs) to help reduce the risk of contracting the virus and transmitting it to vulnerable patients, especially older adults in residential care facilities. Vaccination uptake among HCWs remains low.
\end{abstract}

Aim To investigate HCWs' attitudes towards, and beliefs about, seasonal influenza vaccination in a residential care facility for older adults in the Republic of Ireland.

Method Data were collected using a self-administered questionnaire.

Results A total of 95 questionnaires were distributed, and 35 (37\%) HCWs completed and returned them. During the 201617 flu season, $20(57 \%)$ respondents were vaccinated. Primary predictors of vaccination acceptance were the belief that being healthy should not mitigate against requiring the vaccine $(r=0.7, P=0.01)$, protection of self and family $(r=0.67$, $P=0.01)$, protection of patients $(r=0.592, P=0.01)$ and agreement with mandatory vaccination $(r=0.351, P=0.039)$. Reasons for vaccination avoidance were misconceptions about the need for vaccination among healthy HCWs $(67 \%)$, efficacy of the vaccine $(60 \%)$, lack of trust in the vaccine $(47 \%)$ and a belief that the vaccine may cause the flu $(47 \%)$.

Conclusion Addressing HCWs' beliefs relating to the personal benefits of vaccination while simultaneously correcting misconceptions may help to increase uptake among those working in residential care settings for older adults.

\section{Author details}

Carmel Halpin, health promotion officer, Health Service Executive Dublin North East, Dublin, Republic of Ireland; Bernie Reid, lecturer, School of Nursing, University of Ulster, Northern Ireland

\section{Keywords}

care homes, infection, influenza, older people, quantitative research, vaccines, viral infections

Seasonal influenza is an acute viral infection that can be responsible for annual epidemics during the winter season across the world. Influenza illness is a preventable infectious disease that is spread from one person to the next through droplets from sneezing, coughing or touching contaminated objects (Centers for Disease Control and Prevention (CDC) 2018). It causes significant morbidity and mortality, and contributes to or causes more deaths globally than all other vaccine-preventable diseases combined (Haridi et al 
2017). Vaccination can help reduce morbidity and mortality among all groups, but it is especially important for people at higher risk of serious complications, including older adults and those who are immunocompromised (Kunisaki and Janoff 2009, Haridi et al 2017).

Residential care facilities accommodate older adults for months and often years in congregate settings. Older adults in such facilities are susceptible to the acquisition and spread of influenza due to their overall frailty, close quarter living arrangements and sharing of caregivers, as well as opportunities for introduction of infection through resident transfers, and the movement of staff and visitors through facilities (Lansbury et al 2017).

Despite high levels of vaccination uptake, low vaccine efficacy in older adults exacerbates their vulnerability to influenza and its potential complications (Daugherty et al 2015). Outbreaks of influenza caused by influenza A and B viruses are well documented in residential care facilities, with high rates of mortality and morbidity, highlighting the need for early recognition and initiation of preventative measures (Hayward 2017, Lansbury et al 2017).

Exposure of older adults in residential care facilities to influenza increases their risk of respiratory-origin hospitalisation and death due to a respiratory cause compared with unexposed residents (Gaillat et al 2009, Hayward 2017). Modelling studies suggest that the burden on healthcare services is particularly onerous for those aged 75 or more, with an estimated $36 \%$ of all influenza-attributable respiratory hospitalisations and 74\% of all influenza-associated deaths occurring in this age group in the UK over 13 seasons (Matias et al 2016).

In view of the potential for healthcare workers (HCWs) to contribute to influenza transmission among older adults in residential care settings (Lu et al 2013), vaccination among HCWs can be a critical second level of defence (Hayward 2017). The European Centre for Disease Prevention and Control (2016) and the World Health Organization (WHO) (2018) [Q1. Is the website provided for this correct?] contend that vaccination remains the most effective single public health intervention to mitigate and prevent influenza spread. In accordance with such recommendations, Ireland's Health Service Executive (HSE) offers the seasonal influenza vaccine free to all HCWs to prevent spread of the infection and to reduce work-related sick leave (Nicoll and Sprenger 2013).

Despite vaccination recommendations and the risks posed by influenza, however, vaccine uptake remains consistently low across European countries (Blank et al 2009, Durando et al 2016). In Ireland, where vaccination is recommended but not mandatory, the uptake rate among hospital HCWs was 32\% for the 2016-17 season. The highest uptake was among medical and dental professionals and the lowest among nursing and other patient and client care staff [Q2. Note: sentence moved to reflect report] (O'Lorcain et al 2017).

In long-term care facilities, vaccine uptake varied by HCW staff category, ranging from $20-45 \%$ with an average uptake of $28 \%$. The highest uptake was among health and social care professionals and the lowest among nursing staff [Q3. Is this put correctly?] (O'Lorcain et al 2017). These figures reflect the gradual increase in uptake rates from previous years, but still fall below the national recommended level of $40 \%$ (Department of Health/HSE 2015) and the international recommended level of 70\% [Q4. Is this figure cited in the reference?] (WHO 2018).

In Northern Ireland, the overall vaccine uptake by HCWs for the 2016-17 season was 29\% (Public Health Agency 2017), while an uptake rate of 69\% for the 2017-18 season was reported in England (Public Health England 2018). By contrast, in the US, where some states have mandatory vaccination, the uptake rate for the 2016-17 season was 79\% (Black et al 2016).

The strongest predictor of HCWs' intention to receive the influenza vaccine is attitude (Lehmann et al 2015), which is in turn moderately to strongly correlated with beliefs, such as a belief or lack of belief in the scientific evidence for vaccine effectiveness (Lehmann et al 2015). Other studies emphasise the centrality of HCWs' attitudes and beliefs as predictors of their intention to receive the vaccine. For example, a positive association has been reported between acceptance of the vaccine and: self-protection motives; willingness to protect patients, family members and/or colleagues; the perception that vaccination is the norm [Q5. Is this put correctly?]; perceived moral obligations to be vaccinated; and the capacity to receive the vaccine conveniently (Anikeeva et al 2009, Hollmeyer et al 2009, Aguilar-Díaz et al 2011, Cohen and Casken 2012).

An Irish study by Muhammad et al (2015) reports that the arrival of the H1N1 influenza pandemic had a significant effect on HCWs' attitudes about receiving the vaccine. Reasons for non-vaccination include low perceived susceptibility of contracting influenza, low perceived severity of influenza, and a lack of belief in the relevance of influenza vaccination and the supporting scientific evidence (Hollmeyer et al 2009, Aguilar-Díaz et al 2011, Cohen and Casken 2012).

Hakim et al (2011) found that HCWs' perceptions about violation of freedom of choice and personal autonomy were reported reasons for opposition to the vaccine. Muhammad et al (2015) found that external factors, specifically media influence, played a significant role in HCWs' decisions to accept or decline the vaccine. Lehmann et al (2015) report that past vaccination uptake explains 
a considerable amount of variance in HCWs' intention to receive the influenza vaccine. In addition, demographic factors, such as being older or a physician rather than a nurse, were associated with higher acceptance of influenza vaccination (Hollmeyer et al 2009 , Aguilar-Díaz et al 2011). Although valuable, research into HCWs' attitudes towards, and beliefs about, influenza vaccination has been conducted primarily in acute hospital settings, and there is a paucity of research conducted in residential care facilities.

\section{Aim}

The aim of the study was to investigate HCWs' attitudes towards, and beliefs about, seasonal influenza vaccination in a residential care facility for older adults.

\section{Method \\ Study design}

The study adopted a descriptive design using a self-administered questionnaire.

\section{Study location}

The study was conducted in one publicly funded residential care facility for older adults in the Republic of Ireland. The facility provides short or long-term nursing or personal care for about 50 residents.

\section{Sampling and recruitment}

A universal sampling method was adopted wherein all HCWs employed in the facility were invited to participate in the study. This type of non-probability sampling was selected because the population of HCWs was small and so the possibility of making analytical generalisations about it was limited [Q6. Is this put correctly?]. For the purposes of the study, HCWs were defined as people who deliver healthcare either directly, as nurses, allied healthcare professionals and healthcare assistants (HCAs), or indirectly, as administration, housekeeping, catering, portering or maintenance staff.

The researcher $(\mathrm{CH})$ provided information sessions for HCWs to outline the purpose and nature of the study. The nurse manager facilitated the distribution and collection of questionnaires. Participation was voluntary and return of a completed questionnaire was considered to indicate implied consent. HCWs were requested to place completed questionnaires in sealed boxes located in staff dining areas. The researcher visited the facility on two further occasions over a four-week period to encourage participation.

\section{Questionnaire items}

A self-administered questionnaire was designed after a review of existing research (Anikeeva et al 2009, Hollmeyer et al 2009, Aguilar-Díaz et al 2011, Cohen and Casken 2012, Lehmann et al 2015, Muhammad et al 2015). The questionnaire comprised three sections:

1. Three items designed to gather demographic information about respondents.

2. Four items inquiring about respondents' vaccination status and reasons for accepting or declining vaccination.

3. A total of 14 positively and negatively worded statements to assess respondents' attitudes and beliefs about influenza and vaccination. Each statement was accompanied by five Likert response options. Positively worded statements could be scored between 1 and -5, where 1 means 'strongly disagree' and 5 means 'strongly agree'; negatively worded statements could be scored between -1 and -5 , where -1 means 'strongly agree' and -5 means 'strongly disagree'. Scores of 3 or -3 indicated ambivalence about the statement of interest [Q7. Is this section put correctly?].

\section{Data analysis}

Data were analysed using Statistical Package for Social Sciences (SPSS) Version 24 (IBM Corporation 2016). Data pertaining to demographic information, vaccination status and reasons for accepting or declining vaccination were explained using descriptive statistics. In addition, mean scores were calculated for each statement relating to attitudes and beliefs. Pearson correlations were used to examine relationships between respondents' attitudes and beliefs, demographic characteristics, and vaccine uptake. This statistical test was deemed appropriate because the variables being studied were normally distributed.

\section{Ethical considerations}

The study protocol was approved by the relevant ethics committees at Ulster University and the HSE in Ireland.

\section{Results}


A total of 95 questionnaires were distributed to HCWs in the residential care setting, with 35 (37\%) responding. The demographic characteristics of HCWs who participated in the study are outlined in Table 1. Most respondents were HCAs $(n=12,34 \%)$, followed by nurses $(n=9,26 \%)$ and housekeeping staff $(n=6,17 \%)$ (Table 1$)$. Almost two thirds of participants were aged 51-64 $(n=23,66 \%)$, and $21(60 \%)$ had been in role for more than 11 years.

\begin{tabular}{|c|c|c|c|}
\hline Variable & & $n$ & $\%$ \\
\hline \multirow[t]{7}{*}{ Role } & Healthcare assistant & 12 & 34 \\
\hline & Nurses & 9 & 26 \\
\hline & Housekeeping staff & 6 & 17 \\
\hline & Catering & 3 & 9 \\
\hline & Management & 2 & 6 \\
\hline & Allied healthcare professionals & 2 & 6 \\
\hline & Administration & 1 & 3 \\
\hline \multirow{3}{*}{$\begin{array}{l}\text { Age } \\
\text { (years) }\end{array}$} & $18-35$ & 2 & 6 \\
\hline & $36-50$ & 10 & 29 \\
\hline & $51-64$ & 23 & 66 \\
\hline \multirow{5}{*}{$\begin{array}{l}\text { Length of } \\
\text { time in } \\
\text { current } \\
\text { role }\end{array}$} & 6 months-2 years & 3 & 9 \\
\hline & 3-5 years & 4 & 11 \\
\hline & $6-10$ years & 7 & 20 \\
\hline & $11-15$ years & 6 & 17 \\
\hline & $>16$ years & 15 & 43 \\
\hline
\end{tabular}

\section{Immunisation status}

More than half of participants $(n=20,57 \%)$ reported having received the influenza vaccine during the 2016-17 season. Vaccine uptake was lower among nurses $(n=5,56 \%)$ and HCAs $(n=5,42 \%)$ than among other staff groups, such as housekeeping $(n=5,83 \%)$ and catering $(n=3,100 \%)$ staff. However, the relationship between vaccine uptake and HCW role was not significant $(r=0.061$, $P=0.364)$. Similarly, there was no relationship between vaccine uptake and age $(r=-0.216, P=0.212)$. For those respondents who received the vaccine, public health workplace clinics were identified as the primary location of vaccination $(n=13,65 \%)$, followed by GP clinics $(n=4,20 \%)$.

Respondents reported that their main reasons for accepting the vaccine were to protect their own families $(n=17,85 \%)$, avoid sick leave $(n=17,85 \%)$, avoid getting the flu $(n=16,80 \%)$ and protect patients in their care $(n=16,80 \%)$ (Table 2$)$. Less influential reasons for accepting the vaccine were doctors' recommendations $(n=9,45 \%)$ and line managers' suggestions $(n=12,60 \%)$.

Conversely, the main reasons for declining vaccination $(n=15,43 \%)$ were respondents' perceptions that they did not need the flu vaccine $(n=10,67 \%)$, concerns about the effectiveness of the vaccine in preventing flu $(n=9,60 \%)$, beliefs that the vaccine may cause the flu $(n=7,47 \%)$ and lack of trust in the vaccine $(n=7,47 \%)$ (Table 3). Most respondents who declined vaccination appeared to indicate that they were aware of recommendations about $\mathrm{HCW}$ vaccination and that organisational factors, such as clinic times, presented no impediment to vaccination.

\begin{tabular}{|c|c|c|}
\hline Statement & $n$ & $\%$ \\
\hline I wanted to avoid getting the flu & 16 & 80 \\
\hline I wanted to protect my family at home & 17 & 85 \\
\hline
\end{tabular}




\begin{tabular}{l|l|l|}
\hline Doctor recommended flu vaccine & 9 & 45 \\
\hline I wanted to protect the patients I cared for & 16 & 80 \\
\hline I know someone who got sick with the flu & 14 & 70 \\
\hline I don't want to be off sick with the flu & 17 & 85 \\
\hline My line manager suggested I have the vaccine & 12 & 60 \\
\hline Table 3. Factors influencing healthcare workers' \\
\hline decision to decline influenza vaccine (n=15) \\
\hline Statement & $\boldsymbol{n}$ & $\%$ \\
\hline Vaccine gave me the flu & 7 & 47 \\
\hline Side effects of the flu vaccine & 6 & 40 \\
\hline I don't need the flu vaccine & 10 & 67 \\
\hline Not aware that I needed a flu vaccine & 3 & 20 \\
\hline I don't believe the flu vaccine prevents flu & 9 & 60 \\
\hline I forgot to get the flu vaccine this year & 2 & 13 \\
\hline Clinic times did not suit me for vaccination & 1 & 7 \\
\hline I don't trust the flu vaccine & 7 & 47
\end{tabular}

\section{Attitudes and beliefs}

All respondents completed this section, with 30 (86\%) either agreeing or strongly agreeing that flu is a serious illness (mean 4.23, SD 0.910) and that they were at risk of acquiring flu (mean 4.11, SD 0.796) (Table 4). However, differing views emerged about whether flu can be easily treated (mean 2.86, SD 1.115), including uncertainty among some participants $(n=10,29 \%)$. Almost three quarters of participants $(n=26,74 \%)$ either agreed or strongly agreed that vaccines are useful (mean 4.00, SD 0.970$)$, but a slightly smaller proportion $(n=23,65 \%)$ either agreed or strongly agreed that the flu vaccine was effective in protecting against the flu (mean 3.86, SD 1.089). Although $21(60 \%)$ respondents either agreed or strongly agreed that the flu vaccine did not cause the flu (mean 3.49 , SD 0.951), 16 (46\%) were concerned about potential side effects of the vaccine, while others expressed uncertainty $(n=8,23 \%)($ mean 2.76, SD 1.046).

\section{Table 4. Healthcare workers' attitudes and beliefs about influenza and vaccination $(n=35)$}

\begin{tabular}{|c|c|c|c|c|c|c|c|c|c|c|c|c|}
\hline \multirow[t]{2}{*}{ Statement } & \multicolumn{2}{|c|}{ Strongly agree } & \multicolumn{2}{|c|}{ Agree } & \multicolumn{2}{|c|}{ Unsure } & \multicolumn{2}{|c|}{ Disagree } & \multicolumn{2}{|c|}{$\begin{array}{l}\text { Strongly } \\
\text { disagree }\end{array}$} & \multirow[t]{2}{*}{ Mean } & \multirow[t]{2}{*}{ SD } \\
\hline & $n$ & $\%$ & $n$ & $\%$ & $n$ & $\%$ & $n$ & $\%$ & $n$ & $\%$ & & \\
\hline Flu is a serious illness & 16 & 46 & 14 & 40 & 2 & 6 & 3 & 9 & - & - & 4.23 & 0.910 \\
\hline I am at risk of catching the flu & 11 & 31 & 19 & 54 & 3 & 9 & 2 & 6 & - & - & 4.11 & 0.796 \\
\hline Influenza infection can be easily treated & 4 & 11 & 10 & 29 & 10 & 29 & 9 & 26 & 2 & 6 & 2.86 & 1.115 \\
\hline I believe that vaccines are useful & 12 & 34 & 14 & 40 & 7 & 20 & 1 & 3 & 1 & 3 & 4.00 & 0.970 \\
\hline $\begin{array}{l}\text { Getting the flu vaccine is a good way to } \\
\text { protect against catching the flu }\end{array}$ & 12 & 34 & 11 & 31 & 8 & 23 & 3 & 9 & 1 & 3 & 3.86 & 1.089 \\
\hline The flu vaccine itself does not cause flu & 3 & 9 & 18 & 51 & 8 & 23 & 5 & 14 & 1 & 3 & 3.49 & 0.951 \\
\hline $\begin{array}{l}\text { I have concerns about the side effects of the } \\
\text { flu vaccine }\end{array}$ & 3 & 9 & 13 & 37 & 8 & 23 & 9 & 26 & 2 & 6 & 2.76 & 1.046 \\
\hline I am healthy and I don't need the flu vaccine & 4 & 11 & 10 & 29 & 5 & 14 & 12 & 34 & 4 & 11 & 3.06 & 1.277 \\
\hline $\begin{array}{l}\text { Getting the flu vaccination not only protects } \\
\text { you, but your patients }\end{array}$ & 13 & 37 & 13 & 37 & 5 & 14 & 3 & 9 & 1 & 3 & 4.16 & 0.969 \\
\hline $\begin{array}{l}\text { Getting the flu vaccination not only protects } \\
\text { you, but also your family }\end{array}$ & 14 & 40 & 10 & 29 & 5 & 14 & 4 & 11 & 2 & 6 & 3.94 & 1.153 \\
\hline
\end{tabular}




\begin{tabular}{|c|c|c|c|c|c|c|c|c|c|c|c|c|}
\hline $\begin{array}{l}\text { I am aware that vaccination against flu is } \\
\text { recommended for all healthcare workers }\end{array}$ & 17 & 49 & 15 & 43 & 2 & 6 & 1 & 3 & - & - & 4.41 & 0.701 \\
\hline The flu vaccine is easily accessible & 10 & 29 & 22 & 63 & 1 & 3 & 1 & 3 & 1 & 3 & 4.11 & 0.832 \\
\hline $\begin{array}{l}\text { My doctor does not recommend the flu } \\
\text { vaccine }\end{array}$ & 2 & 6 & 3 & 9 & 7 & 20 & 18 & 51 & 5 & 14 & 3.68 & 0.944 \\
\hline $\begin{array}{l}\text { The flu vaccination should be mandatory for } \\
\text { all healthcare workers }\end{array}$ & 7 & 20 & 7 & 20 & 5 & 14 & 10 & 29 & 6 & 17 & 2.97 & 1.424 \\
\hline
\end{tabular}

Less than half of respondents $(n=16,45 \%)$ either disagreed or strongly disagreed that being healthy should mitigate against requiring the flu vaccine (mean 3.06, SD 1.277). Nevertheless, there was a strong correlation ( $r=0.7, P=0.01)$ between the belief that being healthy should not mitigate against requiring the vaccine and acceptance of the vaccine. Analysis further revealed that most respondents either strongly agreed or agreed that the flu vaccine protects patients and themselves $(n=26,74 \%)$ (mean 4.16, SD 0.969) and their family ( $n=24,69 \%$ ) (mean 3.94, SD 1.153). Interestingly, the correlation between respondents' acceptance of the vaccine and their desire to protect their families $(r=0.67, P=0.01)$ was greater than the correlation between vaccine acceptance and the desire to protect patients $(r=0.592, P=0.01)$.

More than $90 \%(n=32)$ of respondents were aware that vaccination against the flu was recommended for all HCWs (mean 4.41, SD 0.701 ) and agreed that the vaccine was easily accessible (mean 4.11, SD 0.832). It was acknowledged that the vaccine was advocated by doctors, with 23 (65\%) respondents either disagreeing or strongly disagreeing with the statement 'my doctor does not recommend the flu vaccine' (mean 3.68, SD 0.944). However, the relationship between doctor recommendation and vaccine acceptance was not significant $(r=0.333, P=0.055)$. Only $14(40 \%)$ considered that flu vaccination should be mandatory for all HCWs, with those who accepted vaccination more likely to agree with a mandatory approach $(r=0.351, P=0.039)$.

\section{Discussion}

This study shows that $57 \%$ of HCWs in a residential care facility for older adults received the influenza vaccine during the 2016-17 season. Uptake rates are consistent with global vaccination rates among nursing home staff, of between 50\% and 60\% (CDC 2012), but exceed the average uptake rates of $32 \%$ for HCWs working in hospitals and $28 \%$ for HCWs working in long-term care facilities in Ireland over the 2016-17 season (O'Lorcain et al 2017).

Shahrabani et al (2009) report, however, that past vaccine acceptance does not necessarily indicate future vaccine acceptance. Consequently, strategies to promote vaccination should adopt a holistic, person-centred approach that incorporates a comprehensive understanding of HCWs' attitudes and beliefs about the predictors of, and barriers to, vaccination (Bellia et al 2013).

Although more than half of HCWs in this study were vaccinated, a much greater proportion were aware that flu is a serious illness, that vaccination is an appropriate method of protecting against the flu and that vaccination is recommended for all HCWs. However, misconceptions remained about the need for vaccination among healthy HCWs, the efficacy and trustworthiness of the vaccine, and its possible side effects. These findings are consistent with those of previous studies (Hollmeyer et al 2009, Aguilar-Díaz et al 2011, Cohen and Casken 2012, Haridi et al 2017).

In an investigation of the effect of knowledge on willingness to accept vaccination, Slaunwhite et al (2016) suggest that knowledge is a significant predictor $(P<0.05)$ at organisational and legislative levels, but not at HCW level. However, improved strategies to educate HCWs specifically on influenza and its associated risks can dispel misconceptions and improve vaccination uptake rates (Riphagen-Dalhuisen et al 2012, Bonaccorsi et al 2015).

The important predictors of vaccine uptake in the study were a desire to protect one's self, and a desire to protect one's family and patients. Like other studies, such as those by Hollmeyer et al (2009) and Corace et al (2013), this study suggests that uptake is enhanced by HCWs' perceptions that vaccination has a positive effect by reducing ill-health and absenteeism. Conversely, uptake is discouraged if HCWs perceive a negative effect on themselves. A note of caution is warranted about fear of absenteeism as a predictor of vaccine uptake, however, because many HCWs continue to work despite being ill with influenza, thereby increasing the risk of transmission to patients and colleagues (Kuster et al 2011).

In addition to self-protection, altruism was also significant in HCWs' vaccination decisions. The protection of family and patients were important reasons for accepting vaccination, with family protection being the most significant factor influencing vaccine uptake along with not wanting to be off sick with the flu [Q8. Is the last phrase correct?]. Similarly, Vasilevska et al (2014) and Durando et al (2016) found that HCWs accept vaccination primarily for their own benefit and that of their family, rather than for the benefit of 
patients. However, Anikeeva et al (2009) suggest that HCWs have an obligation to accept influenza vaccination on the grounds of beneficence and non-maleficence, and to act in the best interests of patients at all times. It could therefore be argued that the results of this study highlight an individualistic approach to vaccination in which ethical aspects of protecting patients may be undervalued. Future vaccination campaigns targeting HCWs could therefore consider emphasising personal vaccination benefits while addressing personal vaccination barriers to increase uptake.

Contrary to other studies, such as those by Smedley et al (2007) [Q9. Please provide reference details for Smedley et al 2007 or replace with a newer one] and Lehmann et al (2015), this study found that organisational factors, such as lack of access to vaccination and inconvenient clinic times [Q10. Is this put correctly?], were not perceived by HCWs as barriers to vaccination. In addition, managers were not considered influential on decisions to be vaccinated. Nevertheless, the availability of onsite vaccination clinics and a greater emphasis on identifying staff champions or role models may raise vaccine uptake rates (Lehmann et al 2015). Managerial and leadership support have also been associated with higher vaccine uptake rates (Ajenjo et al 2010).

It is noteworthy that $40 \%$ of $\mathrm{HCWs}$ in this study agreed that vaccination should be mandatory, and that vaccine uptake was associated with a higher level of support for a mandatory vaccination programme. Influenza vaccination is not mandatory in Ireland, but some policymakers in Canada and the US mandate the vaccine for HCWs [Q11. Does this mean vaccination is mandatory in some states but not others?] (Gruben et al 2014).

Studies have pointed to the advantages of mandatory vaccination in terms of high uptake rates, 'herd immunity', cost-effectiveness and sustainability (Ribner et al 2008, Poland 2010, Quan et al 2012). However, mandatory vaccination remains controversial, mainly due to the ethical challenges of 'forcing' HCWs to accept a vaccine that can infringe on their personal liberty (Anikeeva et al 2009, Rakita et al 2010). Hakim et al (2011) found that HCWs' perceptions about violation of freedom of choice and personal autonomy were reported reasons for opposition to the vaccine, while Lukas (2014) reports that mandatory vaccination programmes were badly perceived by European HCWs. A study by Daugherty et al (2015) shows that offering HCWs attractive incentives, such as involvement in a gift card raffle, can be a more viable alternative to mandated acceptance of the vaccine. However, such incentives cost healthcare providers money, which must be factored into healthcare budgets when influenza vaccination campaigns are being planned.

\section{Strengths and limitations}

A major strength of this study is the lack of previous similar studies in residential care facilities for older adults (Apenteng and Opoku 2014). To the best of the authors' knowledge, the study is the first of its kind in Ireland to investigate HCWs' attitudes and beliefs about seasonal influenza vaccine.

Despite the authors' efforts to create and incorporate personal and contextually driven approaches, including gaining gatekeeper support, delivering information sessions, emphasising the relevance of the study to HCWs' practice, designing a questionnaire that was easy to understand and required minimal time to complete, and making multiple contacts with potential participants, the study's response rate, at 37\%, was disappointingly low (Burke and Hodgins 2015). This low response rate introduces the possibility of inclusion bias because the views of included HCWs may differ from those of excluded HCWs, and is therefore a limitation of the study [Q12. Is this put correctly?].

Another limitation is that the sample, being recruited from one residential care facility for older adults, may not be representative of HCWs working in such facilities, thereby limiting the generalisation of the study's results. There is therefore a need for further studies to gain an in-depth understanding of HCWs' knowledge of, beliefs about and attitudes towards influenza vaccination. This will help healthcare providers and policymakers make important decisions that will influence seasonal influenza vaccination.

\section{Conclusion}

Residential care facilities are important settings for the delivery of long-term care for older adults, and their role in healthcare provision is becoming increasingly critical as the population in Ireland and other Western countries ages. In view of the importance of residential care facilities and the increasing vulnerability of older adults to influenza, greater attention should be paid to HCW vaccination in such facilities.

The results of this study provide greater insight into the attitudes and beliefs of HCWs about the seasonal influenza vaccine in one long-term care facility. These results may be taken into consideration to customise and improve any future person-centred vaccination promotion campaigns, emphasise the personal and altruistic benefits of vaccination for HCWs, and overcome misconceptions that may have an adverse effect on vaccine uptake. This type of approach could be more effective than those addressing organisational factors, 
which did not emerge as obstacles to vaccination. Future studies could expand on this work by evaluating the effects of any such promotion campaign.

\section{Implications for practice}

[Q13. Please provide 3 bullet point sentences that are take home messages for readers]

\section{RELATED NURSING OLDER PEOPLE ARTICLES}

The challenges of caring for patients with influenza

rcni.com/flu-care-challenges

Barriers to keeping warm in later life

rcni.com/warmth-barriers

\section{References}

Aguilar-Díaz F, Jiménez-Corona M, Ponce-de-León-Rosales S et al (2011) Influenza vaccine and healthcare workers. Archives of Medical Research. 42, 8, $652-657$.

Ajenjo M, Woeltje K, Babcock $\mathrm{H}$ et al (2010) Influenza vaccination among healthcare workers: ten-year experience of a large healthcare organization. Infection Control and Hospital Epidemiology. 31, 3, 233-240.

Anikeeva O, Braunack-Mayer A, Rogers W (2009) Requiring influenza vaccination for health care workers. American Journal of Public Health. 99, 1, 24-29.

Apenteng B, Opoku S (2014) Employee influenza vaccination in residential care facilities. American Journal of Infection Control. 42, 3, 294-299.

Bellia C, Setbon M, Zylberman P et al (2013) Healthcare worker compliance with seasonal and pandemic influenza vaccination. Influenza and Other Respiratory Viruses. 7, Suppl 2, 97-104.

Black C, Yue X, Ball S et al (2016) Health Care Personnel and Flu Vaccination, Internet Panel Survey, United States, November 2017. www.cdc.gov/flu/fluvaxview/hcpips-nov2017.htm (Last accessed: 22 January 2019.)

Blank P, Schwenkglenks M, Szucs T (2009) Vaccination coverage rates in eleven European countries during two consecutive influenza seasons. Journal of Infection. $58,6,446-458$.

Bonaccorsi G, Santomauro F, Porchia B et al (2015) Beliefs and opinions of health care workers and students regarding influenza and influenza vaccination in Tuscany, Central Italy. Vaccines. 3, 1, 137-147.

Burke M, Hodgins M (2015) Is ‘Dear colleague' enough? Improving response rates in surveys of healthcare professionals. Nurse Researcher. 23, 1, 8-15.

Centers for Disease Control and Prevention (2012) Prevention and control of influenza with vaccines: recommendations of the Advisory Committee on Immunization Practices (ACIP) - United States, 2012-13 influenza season. Morbidity and Mortality Weekly Report. 61, 32, 613-618.

Centers for Disease Control and Prevention (2018) Influenza (Flu). www.cdc.gov/flu/protect/keyfacts.htm\#flu-vaccination (Last accessed: 22 January 2019.)

Cohen D, Casken J (2012) Why are healthcare workers so resistant to the acceptance of influenza vaccine? A review of the literature to examine factors that influence vaccine acceptance. International Journal of Caring Sciences. 5, 1, 26-35.

Corace K, Prematunge C, McCarthy A et al (2013) Predicting influenza vaccination uptake among health care workers: what are the key motivators? American Journal of Infection Control. 41, 8, 679-684.

Daugherty J, Blake S, Grosholz J et al (2015) Influenza vaccination rates and beliefs about vaccination among nursing home employees. American Journal of Infection Control. 43, 2, 100-106.

Department of Health, Health Service Executive (2015) Healthy Ireland in the Health Services. National Implementation Plan 2015-2017. HSE, Dublin.

Durando P, Alicino C, Dini G et al (2016) Determinants of adherence to seasonal influenza vaccination among healthcare workers from an Italian region: results from a cross-sectional study. BMJ Open. 6, 5, e010779.

European Centre for Disease Prevention and Control (2016) Seasonal Influenza Vaccination in Europe - Vaccination Recommendations and Coverage Rates for 201314 and 2014-15. www.ecdc.europa.eu/en/publications-data/seasonal-influenza-vaccination-europe-vaccination-recommendations-and-coverage-0 (Last accessed: 22 January 2019.)

Gaillat J, Chidiac C, Fagnani F et al (2009) Morbidity and mortality associated with influenza exposure in long-term care facilities for dependent elderly people. European Journal of Clinical Microbiology \& Infectious Diseases. 28, 9, 1077-1086. 
Gruben V, Siemieniuk R, McGeer A (2014) Health care workers, mandatory influenza vaccination policies and the law. Canadian Medical Association Journal. 186, 14, 1076-1080.

Hakim H, Gaur A, McCullers J (2011) Motivating factors for high rates of influenza vaccination among healthcare workers. Vaccine. 29, 35, 5963-5969.

Haridi H, Salman K, Basaif E et al (2017) Influenza vaccine uptake, determinants, motivators, and barriers of the vaccine receipt among healthcare workers in a tertiary care hospital in Saudi Arabia. Journal of Hospital Infection. 96, 3, 268-275.

Hayward A (2017) Influenza vaccination of healthcare workers is an important approach for reducing transmission of influenza from staff to vulnerable patients. PLoS One. 12, 1, e0169023.

Hollmeyer H, Hayden F, Poland G et al (2009) Influenza vaccination of health care workers in hospitals: a review of studies on attitudes and predictors. Vaccine. 27, 30 3935-3944.

IBM Corporation (2016) IBM SPSS Statistics for Windows, Version 24.0. IBM Corporation, Armonk NY.

Kunisaki K, Janoff E (2009) Influenza in immunosuppressed populations: a review of infection frequency, morbidity, mortality, and vaccine responses. The Lancet. Infectious Diseases. 9, 8, 493-504.

Kuster S, Shah P, Coleman B et al (2011) Incidence of influenza in healthy adults and healthcare workers: a systematic review and meta-analysis. PLoS One. 6, 10, e26239.

Lansbury L, Brown C, Nguyen-Van-Tam J (2017) Influenza in long-term care facilities. Influenza and Other Respiratory Viruses. 11, 5, 356-366.

Lehmann B, Ruiter R, van Dam D et al (2015) Sociocognitive predictors of the intention of healthcare workers to receive the influenza vaccine in Belgian, Dutch and German hospital settings. Journal of Hospital Infection. 89, 3, 202-209.

Lu P, Singleton J, Euler G et al (2013) Seasonal influenza vaccination coverage among adult populations in the United States, 2005-2011. American Journal of Epidemiology. 178, 9, 1478-1487.

Lukas K (2014) Factors Influencing Healthcare Professionals' Willingness to Be Vaccinated. Poster presentation, 10 ${ }^{\text {th }}$ International Congress of The European Union Geriatric Medicine Society, Rotterdam, 17-19 September. [Q14. Can you provide a web link to the poster?]

Matias G, Taylor R, Haguinet F et al (2016) Modelling estimates of age-specific influenza-related hospitalisation and mortality in the United Kingdom. BMC Public Health. 16, 481 .

Muhammad H, Gueret P, Hayes B (2015) Attitudes of hospital healthcare workers towards influenza vaccination in a tertiary hospital setting. Irish Medical Journal. 108, $6,185-187$

Nicoll A, Sprenger M (2013) Low effectiveness undermines promotion of seasonal influenza vaccine. The Lancet. Infectious Diseases. 13, 1, 7-9.

O'Lorcain P, Cotter S, Kelleher K (2017) Uptake of the Seasonal Influenza Vaccine in Acute Hospitals and Long-Term Care Facilities in Ireland in 2016-2017: A Report by the Health Protection Surveillance Centre. HPSC, Dublin.

Poland G (2010) Mandating influenza vaccination for health care workers: putting patients and professional ethics over personal preference. Vaccine. 28, 36, 5757 5759 .

Public Health Agency (2017) Surveillance of Influenza in Northern Ireland 2016-2017. Public Health Agency, Belfast.

Public Health England (2018) Seasonal Influenza Vaccine Uptake in Healthcare Workers (HCWs) in England: Winter Season 2017 to 2018. PHE, London.

Quan K, Tehrani M, Dickey L et al (2012) Voluntary to mandatory: evolution of strategies and attitudes toward influenza vaccination of healthcare personnel. Infection Control and Hospital Epidemiology. 33, 1,63-70.

Rakita R, Hagar B, Crome P et al (2010) Mandatory influenza vaccination of healthcare workers: a 5-year study. Infection Control and Hospital Epidemiology. 31, 9 , 881-888.

Ribner B, Hall C, Steinberg J et al (2008) Use of a mandatory declination form in a program for influenza vaccination of healthcare workers. Infection Control and Hospital Epidemiology. 29, 4, 302-308

Riphagen-Dalhuisen J, Gefenaite G, Hak E (2012) Predictors of seasonal influenza vaccination among healthcare workers in hospitals: a descriptive meta-analysis Occupational and Environmental Medicine. 69, 4, 230-235

Shahrabani S, Benzion U, Yom Din G (2009) Factors affecting nurses' decision to get the flu vaccine. European Journal of Health Economics. 10, 2, 227-231.

Slaunwhite J, Smith S, Halperin B et al (2016) The role of healthcare provider attitudes in increasing willingness to accept seasonal influenza vaccine policy changes. Vaccine. 34, 47, 5704-5707.

Smedley J, Poole J, Waclawski E et al (2007) Influenza immunisation: attitudes and beliefs of UK healthcare workers. Occupational and Environmental Medicine. 64, 4, 223-227. [Q9. Is this the Smedley reference you meant to use?] 
Vasilevska M, Ku J, Fisman D (2014) Factors associated with healthcare worker acceptance of vaccination: a systematic review and meta-analysis. Infection Control and Hospital Epidemiology. 35, 6, 699-708.

World Health Organization (2018) Influenza (Seasonal). www.who.int/news-room/fact-sheets/detail/influenza-(seasonal) (Last accessed: 22 January 2019.) [Q16. Is this WHO reference the one you meant to use?] 